\title{
A fuzzy computational model of emotion for cloud based sentiment analysis
}

\author{
Charalampos Karyotis ${ }^{1}$, Faiyaz Doctor ${ }^{1}$, Rahat Iqbal ${ }^{1}$, Anne James ${ }^{1}$ and Victor Chang ${ }^{2,3}$ \\ ${ }^{I}$ Faculty of Engineering, Environment and Computing, Coventry University, Priory Street, CV1 5FB,Coventry, UK \\ ${ }^{2}$ School of Computing, Creative Technologies \& Engineering, Leeds Beckett University, Leeds, LS6 3QR, UK \\ ${ }^{3} X i$ 'an Jiaotong Liverpool University, Suzhou, P. R. China
}

karyotic@uni.coventry.ac.uk/ic.victor.chang@gmail.com

\begin{abstract}
This paper presents a novel emotion modeling methodology for incorporating human emotion into intelligent computer systems. The proposed approach includes a method to elicit emotion information from users, a new representation of emotion (AV-AT model), a genetically optimized adaptive Fuzzy Logic technique for modeling this representation, and a framework for predicting and tracking user's affective trajectory over time. The fuzzy technique is evaluated in terms of its ability to model user affective states in comparison to other existing machine learning approaches. The performance of the proposed affect modeling methodology is tested through the deployment of a personalised learning system. A hybrid cloud intelligence infrastructure is used to conduct large-scale experiments to analyze user sentiments and associated emotions, using data from a million Facebook users. A performance analysis of the infrastructure on processing, analyzing, and data storage has been carried out, illustrating its viability for largescale data processing tasks. A comparison of an emotion categorizing approach using this paper's suggested emotion categories, with Facebook's sentiment analysis API demonstrates that our approach can achieve comparable performance. Finally, discussions on research contributions to cloud intelligence on sentiment analysis, emotion modeling, big data science, and comparisons with other approaches are presented in detail.
\end{abstract}

Keywords-hybrid cloud; big data; emotion modeling; affective computing; adaptive fuzzy systems; social network sentiment analysis;

\section{INTRODUCTION}

The modern and technologically advanced realm of interconnected computing artifacts calls for techniques, which enable the surrounding environment to behave in intelligent ways. Ambient Intelligence (AmI) emerged to satisfy this need, by providing intelligence to networks of electronic devices around us [14]. However, to realize the vision of AmI the development of truly intelligent systems calls for a basic understanding of core aspects of human behavior, such as emotions. Emotion is a basic characteristic of human nature, which influences performance, decision-making, and the interpretation, and assimilation of knowledge. Recognizing and representing human emotion is a problem for which mathematical or traditional modelling can be ineffective. This is because the processes are too complex for mathematical reasoning, and containinherent uncertainties pertaining to 
human nature. Nowadays, humans facilitated by the rapid advancements in technology, produce huge quantities of personalized and contextualized affective information. Social networks are a platform where millions of people interact, share opinions, and express their feelings. The development of computational intelligence techniques enables these platforms to become modern large-scale laboratories in which the development of intelligent emotion aware applications can be incubated with the aim of maximising the quality of computerized solutions [9, 51]. Social network sentiment analysis [48] promises to improve the quality offered by products and services, by automatically detecting user opinion, including evaluations and affective state. In order to fulfill its goals, Sentiment Analysis can largely benefit from computational intelligence techniques able to handle the inherent challenges of Big Data and human affect.

Affective Computing (AC) is an emerging interdisciplinary scientific field, which endeavors to develop intelligent machines that incorporate the user's affect into their design, in order to provide a higher level of human-machine interaction. AC attempts to bridge the gap between the highly emotional human and the emotionally challenged computer [8]. Professor Rosalind W. Picard provided the first and the most widely accepted definition of $\mathrm{AC}$, which states that, "Affective computing is computing that relates to, arises from or deliberately influences emotion" $[49,50]$. Considering the fact that it is difficult even for humans to understand and express their own emotions accurately, we can appreciate the great challenge concerning the ability of an artificial entity to successfully recognize and model affect. Hence, the emotion theory utilized towards the development of an application is crucial to the design of the application. This choice influences the affect recognition, and modeling aspects of the system, since different representations favor different configurations, and pose certain limitations. Indeed, some emotion models favor facial recognition, while others favor the utilization of different modalities such as physiology, voice, text etc. As it was stated in [8] a computer application that incorporates affect in its design can never be separated completely from the underlying emotion theory. In [8] Calvo et al. highlight the need for affective computing researchers to understand and contribute in the emotion modeling literature. There are considerable limitations concerning the utilization of different emotion models. In order to overcome these limitations, this paper proposes a new representation of emotion called the AV-AT model of emotion. By utilizing the AV-AT model, an applicable and effective way to incorporate emotion in the design of intelligent computer systems is presented in order to realize affective computing capabilities.

As seen in review studies by Marcella et al. [42] and Kowalcuzk et al. [36], previous 
research attempts to construct computational models of emotion were oriented towards developing virtual agents which generated realistic human-like emotion responses. Attempts to develop computational models of emotion with more affect recognition oriented goals (as the one described in this paper) are rare to non-existent. The authors argue that the development and testing of computational models, under the scope of affectrecognition, will reveal useful basic affect components. Observing and estimating the parameters, or affect dimensions of emotion is a problematic task impacting upon the ability of an AC application to provide accurate emotion labeling. As Calvo states, "Identifying the appropriate level of representation for practical AC applications is still an unresolved question" [8]. In this paper, an emotion representation with a reasonable tradeoff between accuracy and complexity is provided. It utilizes novel dimensional models of emotion under an affective computing scope. This representation is modeled through a genetically optimized adaptive fuzzy logic technique which aims to be accurate, user friendly, not computationally expensive, and to reflect the underlying emotion model. The fuzzy computational mechanism along with the AV-AT model of emotion form the core components of the proposed emotion modeling methodology. This methodology is used in the development of a personalized learning system, which provides a benchmark for other AC applications to utilize the proposed approach.

In this research paper, the authors aim to illustrate the usefulness of implementing the proposed fuzzy emotion representation model, and to demonstrate its effectiveness and applicability in big data settings. The big data settings are represented through a hybrid cloud intelligence infrastructure with sentiment analysis, social network analysis, data queries, and data collected from one million Facebook users. The rest of the paper is organized as follows: in section 2, the authors present the necessary background knowledge regarding different emotion representations. Moreover, the use of fuzzy logic in affect modeling is justified, and evidence is provided to support the use of Education as a suitable context for testing the proposed emotion modeling and categorization methodology. Section 3.1 includes the online survey, which provided the data from which the computational emotion model was created. In section 3.2, the fuzzy rule extraction, optimization, and adaptation method used to construct the data driven fuzzy model is explained in detail. In section 3.3, the performance of our fuzzy emotion modeling approach is tested and validated against other machine-learning approaches. In section 3.4, the implementation of a personalized learning system using this affect modeling approach is presented. In section 3.5, the suggested emotion modeling methodology is evaluated and useful conclusions are extracted, with the help of two experimental tutorial sessions. In 
section 4, a hybrid cloud, involving repository and processing resources at four different sites, is used to evaluate a proposed cloud intelligence service. Moreover, this section includes key functions and the use of sentiment analysis to categorize Facebook users' emotions, based on the set of emotions discussed in this paper. In section 5, large-scale experiments are carried out and the results of processing, analyzing, and storing data are used to support the case of the presented cloud intelligence system. Section 6 discusses the impact of our research, and draws comparisons with other approaches. Finally, in section 7 general conclusions and future research directions are discussed, and our research contributions are summarized.

\section{BACKGROUND}

\subsection{CLASSIFYING EMOTION}

Understanding and classifying emotions is a very complex and delicate task, still under debate among psychologists. In 1884, the American psychologist and philosopher William James wondered: "What is an emotion?" and this question has triggered a discussion, which is still active today [27]. A common answer to this question is that emotions are mental states provided by the structure of the nervous system, which result in consistent and specific patterns of physiology, such as movements of the facial muscles, feelings, and behavior [39]. This approach is congruent with the perception of emotions as "natural kinds", meaning they are distinct, they exist in nature, and they can be identified independently of human perception [39]. An illustration of this kind of emotion modeling approach was in the 1970s when Paul Ekman using cross-cultural facial expressions experiments, identified a set of six basic emotions (anger, disgust, fear, happiness, sadness and surprise, also known as the "Big Six") [25]. This "natural kind" approach can be immediately challenged if we consider the infinite number of states, which should be named as distinct emotions. A major problem for Affective Computing researchers is the fact that emotion labeling is highly dependent on the cultural background of the people under investigation [59], and the context of the application [2]. Hence, it is almost impossible to create the necessary databases to reflect the massive number of emotions, while at the same time account for cultural and contextual differences. Most systems using discrete emotion models are based on facial recognition and due to the limitations described above they are constrained to use sets of emotions, which do not necessarily reflect the affective state of the user. This is highlighted in Zeng et al.'s review, where most of the systems, which relied on facial recognition, used Ekman's Big Six, despite the fact that those emotions were irrelevant to the context of the application [65]. Additionally, as it was 
stated in [22] "basic emotions" have been emphasized in AC systems at the expense of other "non-basic" emotions. The team's results showed that other "non basic" emotions, such as engagement, boredom, confusion, and frustration occurred at a much larger scale after generalizing across tasks, interfaces, and methodologies [22].

Contrary to the view that emotions are natural kinds, psychological constructivism suggests that emotions emerge from the combination of more basic structural elements. These psychological primitives combine in numerous ways in order to produce a variety of mental and affective experiences, such as emotions [46]. For example, a very popular view is that emotions originate from a two dimensional space called core affect [54]. The first dimension is arousal (how passive or active someone is) and the second dimension is valence (how positive or negative someone feels). Through cognitive elaboration, core affect can be converted to emotions [55]. To illustrate this, when someone feels stressed, this can be defined as an affective state of negative valence and high arousal. This arousal valence (AV) representation of emotion is a very popular model used by $\mathrm{AC}$ researchers $[41,56]$. The authors argue that this approach poses limitations in the selection of emotions to describe the user's affective state. The researcher is bound to using emotions easily separable in AV space, otherwise the affect recognition part of their system might underperform. Moreover, the nature and number of the basic structural elements of emotion is still under investigation [18], and as a result, the same applies to the dimensional representation of emotion in AC systems.

A simple and modern approach in emotion modeling is the recently introduced Affective Trajectories hypothesis. According to the AT hypothesis "emotion arises partly from the interaction of the evaluations of one's current state, predictions of the future, and the outcomes that one experiences after these predictions" [17]. These processes interact, and combine with each other to create an emotional experience [35]. For example, anger can emerge when a positive prediction is followed by a negative outcome. A framework for utilizing the AT theory in AC was presented in [33]. There are certain limitations concerning the AT framework since it was previously tested in a context free environment, and despite the fact that these basic cues demonstrated some predictive power, it is necessary for the oversimplified AT hypothesis structure to be enriched in order to be able to differentiate efficiently between different emotion labels. This paper proposes the utilization of a two stage modeling approach, by combining the AV representation of emotion, with the AT hypothesis terms as the AV-AT model. In the first stage, emotion labels emerge from different combinations of the person's prediction about the future, arousal, and valence. In the second stage, emotion labels emerge from different 
combinations of the person's evaluation of the outcome after their predictions, arousal, and valence. As proven for the AT hypothesis [33], each individual utilizes and combines the basic AT elements in a highly personalized way. As a result, the same applies for the proposed AV-AT model since it is an extension of the AT hypothesis. By using the AVAT model, we aim to differentiate more successfully among emotion labels compared to using other emotion models.

\subsection{FUZZY LOGIC FOR EMOTION MODELING}

The notion of emotion and its structural elements is inherently fuzzy and contains uncertainty. As Wu states in [63], emotion is subject to inter and intrapersonal uncertainty. Interpersonal uncertainty concerns the different perceptions and expressions which individuals have about the same emotion, while intrapersonal uncertainty is the uncertainty an individual has about their own emotions at different times or contexts. Fuzzy logic systems have the ability to handle these innate uncertainties [63] and have been used as a means to represent and model affect relations $[34,33]$. Fuzzy logic systems as proposed by Zadeh [64] are also able to represent and model the relations existing in data with interpretable rules, thus allowing knowledge extraction about the domain under investigation. This research aims to elicit the underlying affect relations as part of the emotion modeling approach, therefore fuzzy rules that can be learnt from user data are proposed as a means to represent these affect relations. In addition, adaptive fuzzy systems have been shown to enhance the capabilities of fuzzy models by enabling online adaptation of the model to occur in response to user and environmental changes [23, 24]. They have also been shown to be very efficient at capturing individual differences concerning emotional expression and construction, with the ability to deliver their results without an excessive computational burden [33]. Fuzzy systems' internal parameters can also be optimized with the use of optimization algorithms in order to provide more precise results. For example in [4], a fuzzy logic system for a financial application was optimized with the use of a genetic algorithm. We can conclude from the above, that the use of a genetically optimized adaptive fuzzy system for representing an uncertain and highly personalized emotion model, such as the one proposed in this paper, is a very reasonable choice of approach.

\subsection{EDUCATION AND SOCIAL NETWORKS FOR EMOTION MODELING}

Our proposed affect modeling approach requires an appropriate context in order to be tested. Education is such a context, since emotions correlate very strongly with learning. Emotions, such as confusion, which is an indicator of cognitive disequilibrium, and flow, 
which represents a state of high involvement and interest, can be considered as desired states for a student since they have a positive effect on learning $[15,16]$. Other emotions like boredom and frustration are identified to have a negative correlation with a student's learning and should be avoided [15]. A number of AC systems take this strong relation into consideration in order to promote the wellbeing of the student $[1,28]$. The personalized system proposed in this paper is tested in an educational context, and more specifically in the context of Activity Led Learning (ALL) $[31,61]$ and Problem Based Learning (PBL) [3]. Both pedagogical frameworks are in line with the two-stage structure of the proposed approach, since they are based on discrete activities with start and end points, which can be the points in time when the student's predictions, evaluations and corresponding affective states are acquired $[32,61]$.

A student centered educational context is a logical testing platform for the proposed affect modeling approach. However, its practical application value would be maximized if this methodology were to be applied in a larger context such as social networks. The use of affect detection and sentiment analysis on the huge amounts of data offered by Facebook or Twitter users can be applied towards creating novel applications in educational or other contexts. The importance of sentiment analysis in social networks has been demonstrated in many studies in the past. In [58] the research team's results revealed that incorporating social-network information leads to statistically significant sentiment classification improvements. Another example, more educationally focused, is in [45] where Ortigosa et al. presented a method for sentiment analysis in Facebook and its potential applications in e-learning. Exploiting massive amounts of social network data for sentiment analysis purposes is a challenging process concerning big data analysis, processing, and storage requirements. In this research, a hybrid cloud is used and tested as an infrastructure for integrating the suggested affect modeling approach. The choice to utilize hybrid cloud can be justified because, as discussed in Hashem et al.'s review, cloud computing is a powerful technology able to perform massive-scale complex computing arising from big data [29].

\section{AV-AT METHODOLOGY}

\subsection{DATA COLLECTION - ONLINE SURVEY}

In this section, an overall design of the online survey is outlined. This survey provided the necessary information for the construction of the generic fuzzy rule base to represent the proposed emotion model. Moreover, through the survey, participant specific data were obtained and used to aid in the development of a more personalized system for individual users. During this survey, the authors aimed to explore and model the affect relations 
existing between a set of emotions, which can be reported by a student to describe their affective state, and the basic elements of the AV-AT model. Namely, the emotion labels of 'flow', 'excitement', 'calm', 'boredom', 'stress', 'confusion', 'frustration', and 'neutral' were used, and their relations with 'arousal', 'valence', 'prediction', and 'evaluation of the outcome' were explored. The survey was conducted with the help of the online tool QuestionPro. Eighty participants of various ethnic origins completed the online survey. All the participants were provided with the necessary instructions for successfully completing the survey. Before proceeding, the participants provided their consent and some basic demographic information (age and gender). The survey was in line with the design proposed by Kirkland et al. [35]. In our research, this design was modified to suit an educational context.

In the online survey, different scenarios specifically designed to induce different combinations of the basic AV-AT elements, were presented to the participants. The scenarios narrated common, education related situations, since our aim was to induce education related emotions. A total of 18 scenarios were presented in a random order. Each scenario consisted of two stages, and asked the participants to imagine themselves in the depicted story. In the first stage, the beginning of the story was presented, and the overall current state, and prediction about the future were described. At this point, the participants were asked to use the sliders provided, and rate their arousal, valence and prediction about the future on a scale of 0 to 100. Prediction ranged from 0 (very negative prediction) to 100 (very positive). Valence ranged from 0 (unpleasant) to 100 (pleasant). Arousal ranged from 0 (deactivated, low arousal) to 100 (activated, high arousal). After providing their prediction, valence and arousal values, the participants were also asked to use sliders in order to rate their affective state for that part of the story. More specifically, the participants were asked to choose from a list of 8 emotions (flow, excitement, calm, boredom, stress, confusion, frustration, and neutral) the extent to which these labels described their affective state. The ratings for these ranged from 0 (not at all) to 100 (perfectly). The participants were free to rate as many of the emotion labels as they wished. In the second stage, the outcome of the story was presented to the participants. During the second stage the participants were asked to rate the outcome of the story, which could be 'worse', 'better' or 'as they had predicted' in the first stage, ranging from 0 (worse than expected, terrible) to 100 (better than expected, great). Then they provided values for the valence and arousal elements along with the target emotions felt. In order to retain the cognitive bond with the previous part of the story and reduce the cognitive load for the participant, the beginning of the story was displayed on the screen to allow them to recap the scenario. 


\subsection{FUZZY MODELING}

In this section, the stages of our fuzzy set and fuzzy rule extraction method are described, along with the optimization and adaptation approaches. This process resulted in the construction of two fuzzy classification systems, one for each stage of the emotion model. The required data in order to construct the computational model were provided from the online survey described in the previous section. The training samples contain 3 inputs and 8 outputs for each stage. In the first stage, the inputs are arousal, valence, and prediction, and in the second stage, they are arousal, valence, and outcome. In both stages, the outputs are values of the eight emotions (flow, excitement, calm, boredom, stress, confusion, frustration, and neutral). All variables take values in the interval $[0,100]$. Every training sample is in the form of $\left(\mathrm{x}^{(\mathrm{ts})} ; \mathrm{y}^{(\mathrm{ts})}\right)$ where ts $=1, \ldots .1440$ since the data collected from the survey is a total of 1440 samples.

\subsubsection{FUZZY SET AND FUZZY RULE EXTRACTION}

Initially, we opted to construct the necessary fuzzy sets from the user survey data to describe the basic elements of prediction, valence, arousal, outcome, and the eight aforementioned emotions. A partitioning of five fuzzy sets was chosen in order to cover the input and output space, so that the extracted model is accurate, but at the same time retains a satisfying degree of interpretability. The Fuzzy C-Means (FCM) clustering algorithm [5] was used in order to define five original fuzzy sets. These fuzzy sets have triangular membership functions. Every membership function has a degree of membership equal to 1 at the center previously calculated by the FCM, and a support that is defined as the space between the projections of the previous center and the next center on the horizontal axis. At this point, it should be noted that the properties of our fuzzy sets are only dependent on the position of their centers. This attribute is later used for the optimization of the system.

After the initialization of the fuzzy sets, a fuzzy rule base is extracted from the data with the help of an enhanced version of the Wang Mendel (WM) method as presented in [60]. Initially, we defined five original fuzzy sets, which covered all inputs and outputs. Let $I_{\text {in }}^{q}$ be the corresponding fuzzy set for the input in $=1, \ldots, 3$ and $G_{\text {out }}^{p}$ be the corresponding fuzzy set for output out $=1, \ldots, 8$ where $q$ and $p=1, \ldots, 5$. The rules we aimed to extract from the data would be in the following form:

$$
\text { If } x_{1} \text { is } I_{1}^{q} \text { and... } x_{3} \text { is } I_{3}^{q} \text { then } y_{1} \text { is } G_{1}^{p} \text { and...and } y_{8} \text { is } G_{8}^{p}
$$

Below we present the proposed method for one of the emotion outputs, as the same applies for the multi-output case.

For each sample $\left(\mathrm{x}^{(\mathrm{ts})} ; \mathrm{y}^{(\mathrm{ts})}\right)$ the membership values $\mu_{\mathrm{F}_{\mathrm{in}}^{\mathrm{q}}}\left(\mathrm{x}_{\text {in }}^{(\mathrm{ts})}\right)$ were calculated for all 
inputs and all corresponding fuzzy sets. Then we proceeded with finding the highest membership value at q́

$$
\mu_{I_{i n}^{q}}\left(x_{i n}^{(t s)}\right) \geq \mu_{I_{c}^{q}}\left(x_{i n}^{(t s)}\right)
$$

for $\mathrm{q}=1, \ldots, 5$. Each sample $\left(\mathrm{x}^{(\mathrm{ts})} ; \mathrm{y}^{(\mathrm{ts})}\right)$ was used in order to extract the following rule:

$$
\text { If } x_{1} \text { is } I_{1}^{\dot{q}} \text { and } \ldots x_{n} \text { is } I_{n}^{q} \text { then y is centered at } y^{(t s)} \text {. }
$$

The weight $\mathrm{w}^{(\mathrm{s})}$ of the rule was also calculated as:

$$
w^{(s)}=\prod_{i n=1}^{3} \mu_{I_{i n}^{q}}\left(x_{i n}^{(t s)}\right)
$$

At this point of the algorithm, every sample was converted to a fuzzy rule. Following this process all the rules with the same If-part were accumulated into a group. Let $\mathrm{W}$ be the number of groups. If we assume that in group g belong $N_{g}$ samples $\left(t_{u}^{g}\right) u=1, \ldots, N_{g}$ consequently, we extract $\mathrm{N}_{\mathrm{g}}$ rules in the form:

$$
\text { If } x_{1} \text { is } I_{1}^{\left(q^{g}\right)} \text { and } \ldots x_{3} \text { is } I_{3}^{\left(q^{g}\right)} \text { then y is centered at } y^{\left(t s_{u}^{g}\right)}
$$

We computed the weighted average using the following formula:

$$
a v^{(g)}=\frac{\sum_{u=1}^{N g} y^{\left(t s_{u}^{g}\right)} w^{\left(t s_{u}^{g}\right)}}{\sum_{u=1}^{N g} w^{\left(t s_{u}^{g}\right)}}
$$

After calculating the membership value for all output fuzzy sets, we selected the fuzzy set with the highest value. Let $p$ be the corresponding set.

$$
\mu_{G} \dot{p}\left(a v^{(g)}\right) \geq \mu_{G} p\left(a v^{(g)}\right)
$$

At the end of this process, the rules contained in that group were merged into a final rule.

$$
\text { If } x_{1} \text { is } I_{1}^{g} \text { and } \ldots x_{3} \text { is } I_{3}^{g} \text { then } \mathrm{y} \text { is } G^{g} \text {. }
$$

where $\mathrm{G}^{\mathrm{g}}$ is the set with the highest membership as identified before.

The fuzzy rule bases extracted were then used by two classification systems. The first classifier was responsible for mapping values of prediction, valence, and arousal to values of the eight emotions. The second classifier mapped values of outcome, valence, and arousal to values of the aforementioned emotions. Both these classifiers utilized product inference, singleton fuzzification, and center average defuzzification to deliver results for stage 1 and 2 of the proposed emotion model respectively. If we consider the final rule base to include a total of $\mathrm{L}$ rules, the output was calculated using the following formula (where $y_{\text {center }}^{(\mathrm{g})}$ is the center of the fuzzy set $\mathrm{G}^{\mathrm{g}}$ ). It is important to notice that the output values were only dependant by the position of the fuzzy set center points.

$$
y=\frac{\sum_{g=1}^{L} y_{c e n t e r}^{(g)}\left(\prod_{i n=1}^{3} \mu_{G_{i n}^{(g)}}\left(x_{i n}\right)\right)}{\sum_{g=1}^{L}\left(\prod_{i n=1}^{3} \mu_{G_{i n}^{(g)}}\left(x_{i n}\right)\right)}
$$




\section{2.2 OPTIMIZATION}

The extracted fuzzy set and rules, as well as the calculation of the output emotions are dependent on the position of the fuzzy set's center points. A genetic algorithm (GA) was applied, in order to optimize the performance of the constructed system. The performance of the system was evaluated in terms of the Normalized Mean Square Error (NRMSE), which was generated based on a validation set. The validation set comprised of data from the online survey, which was set aside and not used in the training of the classification system. Using the GA, we optimized the values for all input and output fuzzy set centers to produce the minimum value for the NRMSE error. Hence, the objective function of the genetic algorithm was defined as the value of the NRMSE calculated in the validation set. Moreover, we also required the results to be interpretable. The center points extracted from the optimization process should lead to a reasonable interpretation, and facilitate the visualization of the affect relations existing in the AV-AT emotion model.

As mentioned in the comparative study conducted by Elbetagi et al., there are four basic parameters affecting the performance of the GA: population size, number of generations, crossover, and mutation rates [26]. In order to achieve a good trade-off between performance and interpretability, a number of different combinations of the aforementioned parameter settings were tested. As a result, the parameter values that were selected generated a small NRMSE, while at the same time the fuzzy set center points corresponded to separate fuzzy terms being represented. As a result, values of parameters that generated non-interpretable results were rejected, since they did not promote an understanding of the proposed AV-AT emotion representation approach. For example, when the GA used a larger population, it generated marginally better results for the NRMSE, nevertheless the fuzzy set center points were not enabling the construction of an interpretable fuzzy rule base. The GA was implemented by utilizing Matlab's optimization toolbox. In order to use this implementation we provided the algorithm with the parameters shown in figure 2. These parameters generated the most desirable results. The optimization process was performed for 55 variables ( 5 center points for each of the 5 fuzzy sets describing 3 input and 8 output variables). The same procedure was repeated for both stages of the emotion model and it included the following steps:

- Divide the data set to a training and a validation set.

- The chromosome representing each individual of the population is defined in terms of the position of the fuzzy set center points, for every input and output. 
- Initialize the original population (20 individuals) to populate the first generation of the genetic algorithm. In the initial population, the original center points calculated by the FCM are also included as individuals.

- Utilize the method described in the previous section in order to build a fuzzy rule-base for each individual of the population with the help of the training data. Create an instance of the classification system based on the extracted rule base. Calculate the classification accuracy of each fuzzy classifier in the validation set.

- The GA uses the population and the objective function values to produce a new population.

- The selection function of the GA, which chooses the parents for the next generation, is set to be the stochastic uniform function.

- Two individuals of the current generation are guaranteed to survive to the next generation, $80 \%$ of individuals of the next generation is produced due to crossover, and the remaining $20 \%$ is produced due to mutation.

- The crossover function combines two individuals from the current generation to create a child for the next generation. In our case, the crossover function creates a random binary vector and selects the genes from the first parent where the vector is 1 and the genes from the second parent where the vector is 0 and then combines them in order to construct to the child.

- The Gaussian function is chosen as the mutation function. A random number extracted from a Gaussian distribution is added to each vector entry of an individual. Through these small changes, the necessary genetic diversity is provided and the GA is able to search a larger space.

- The GA evolves until there is no considerable change in the fitness function $\left(\mathrm{e}^{-6}\right)$ for a consecutive number of generations or until it reaches the maximum number of iterations (100).

- The values of the fuzzy sets' center points for the best individual in the last generation of the GA are used in the construction of the rule base.

The optimization process succeeded at providing a solution that contained a combination of interpretable center points for the fuzzy sets of the proposed model, while at the same time it improved the classification performance of the original fuzzy model that used the center points calculated by the FCM. By utilizing Matlab's 2016 optimization toolbox a basic comparison between the GA and other available optimization techniques was also performed based on classification accuracy of the generated fuzzy classifiers and parameter interpretability. More specifically, we utilized the pattern search (direct search), 
particleswarm (particle swarm), and simulannealbnd (simulated annealing) options provided by the toolbox. The NRMSE results presented in figure 2 justify the utilization of the proposed GA based approach in terms of achieving a marginally better performance error compared to the other algorithms. In figure 2 we can observe that the GA optimized fuzzy set center points for the prediction, arousal, and valence elements offer an interpretable solution. In contrast, other methods provided solutions with lower interpretability. These comparisons are by no means exhaustive but justify the design choices that were made for tuning the fuzzy classifiers parameters.

(a) GA parameters

\begin{tabular}{cc}
\hline \multicolumn{2}{c}{ GA parameters } \\
\hline Number of Variables & 55 \\
Lower Bound, Upper Bound & $-10,110$ \\
Population Type & Double Vector \\
Population Size & 20 \\
Creation Function & Constraint Dependant \\
Fitness scaling & Rank scales \\
Selection Function & Stochastic Uniform \\
Elite Count & 2 \\
Crossover fraction & 0.8 \\
Mutation function & Gaussian \\
Crossover function & Scattered \\
& Function tolerance $e^{-6}$, \\
Stopping Criteria & Stall generations 10, \\
& Max generations 100 \\
\hline
\end{tabular}

(b) Optimization Performance

\begin{tabular}{llllll}
\hline NRMSE & $\begin{array}{l}\text { No } \\
\text { optimization }\end{array}$ & GA & $\begin{array}{l}\text { Pattern } \\
\text { Search }\end{array}$ & $\begin{array}{l}\text { Simulated } \\
\text { Annealing }\end{array}$ & $\begin{array}{l}\text { Particle } \\
\text { Swarm }\end{array}$ \\
\hline Stage 1 & 18.80 & 17.48 & 17.74 & 18.09 & 17.80 \\
Stage 2 & 20.60 & 19.36 & 19.41 & 19.80 & 19.40 \\
\hline
\end{tabular}

(c) Fuzzy center points

\begin{tabular}{ccc}
\hline Prediction & Arousal & Valence \\
\hline$-0,109$ (Very negative) & $-0,059$ (Very low) & $-1,637$ (Very negative) \\
23,986 (Negative) & 25,182 (Low) & 27,430 (Negative) \\
49,442 (Neutral) & 48,088 (Neutral) & 50,842 (Neutral) \\
74,083 (Positive) & 73,399 (High) & 76,422 (Positive) \\
100,871 (Very Positive) & 100,249 (Very High) & 100,479 (Very Positive) \\
\hline
\end{tabular}

Fig.2. (a) GA parameters (b) Optimization performance (c) Fuzzy centers for prediction, arousal, and valence (stage1).

\subsubsection{ADAPTATION}

The adaptation mechanism of the proposed method is a modification of the Adaptive OnLine Fuzzy Inference System (AOFIS) [23] as presented in [33]. This method was exploited in two ways. Firstly, the data samples collected from the responses of a particular user in the online survey (section 3.1) were presented one by one to the system. The system considered them as desired changes provided by the user, and made the necessary changes to the rule base of each classifier. This allowed our method to have a personalized rule base reflecting the user's preferences right from the start, before they engage actively with a realtime version of the system (offline adaptation). Secondly, when the user is utilizing the online version of the system and they are not happy with the results provided to them, they are able to provide their own values of the output emotions, so that the system makes the necessary corrections (online adaptation). In both cases, the adaptation process is as follows. When a new data sample $\left(x^{(t s)} ; y^{(t s)}\right)$ is provided to the system, the membership values $\mu_{I_{i n}^{q}}^{q}\left(x_{c}^{(t s)}\right)$ for all inputs $i n=1, \ldots 3$ and all fuzzy sets $q=1, \ldots, 5$ were computed. The rules that fired are detected, and the rule with the maximum activation value is identified. Assuming $\mathrm{R}$ is the total number of activated rules, by using the following formula we calculated the center points' optimal position $\left(y_{\text {optc }}\right)$, by taking into account the 
contribution of the other R-1 rules that fired.

$$
y_{\text {optc }}=\frac{y^{(t s)}\left(\sum _ { g = 1 } ^ { R } \left(\prod_{i n=1}^{3} \mu_{G_{i n}^{(g)}}\left(x_{i n}^{(t s)}\right)-\sum_{g=1}^{R-1} y_{c e n t e r}^{(g)}\left(\prod_{i n=1}^{3} \mu_{G_{i n}^{(g)}}\left(x_{i n}^{(t s)}\right)\right)\right.\right.}{\prod_{i n=1}^{3} \mu_{G_{i n}^{(g)}}\left(x_{i n}^{(t s)}\right)}
$$

The calculated value is used in order to find the fuzzy set with the highest membership value.

$$
\mu_{G} \dot{p}\left(y_{o p t c}\right) \geq \mu_{\mathrm{G}^{\mathrm{p}}}\left(\mathrm{y}_{\text {optc }}\right)
$$

Ultimately, the highest activation value rule consequent is replaced by the corresponding fuzzy set $\mathrm{G}^{\dot{p}}$.

\subsection{OFFLINE PERFORMANCE COMPARISON}

In order to test the effectiveness of the proposed fuzzy method, the survey data were used to compare the fuzzy method's results with the results provided from different classification systems. This comparison was done for both stages of the emotion model. In the first stage, the inputs were prediction, valence, arousal, and the outputs were the values of the eight targeted emotions. In the second stage, the inputs were the evaluation of the outcome, valence, and arousal and the outputs were the values of the emotion labels. The suggested fuzzy method was compared to a Multilayer Perceptron (MLP) using a single hidden layer containing ten nodes, a Radial Basis Function Network (RBF) using the softmax activation function, a linear regression model (LNR) and a regression tree (RT). The comparison was drawn in terms of the NRMSE, and the ability of each system to identify the dominant emotion (which was considered to be the emotion for which the participant or the system provided the highest value). The comparisons for each stage were performed using ten-fold cross validation. Additionally, in order to compare the AV-AT model with the AV representation of emotion, the NRMSE and dominant emotion accuracy for all evaluated systems are provided, when the systems were trained by using only the arousal and valence values. To identify the dominant emotion we simulated the decision an affective computing researcher would make if they used the AV model, by constructing a minimum distance classifier (D). In order to do this we used the Affective Norms for English Words (ANEW) [6] database to define clusters in arousal-valence space representing each of the eight emotion words. The center of each cluster was the arousal and valence values for this word in the database. Using the arousal and valence values provided by the participant, the Euclidian distances from each clusters' centers were calculated. The minimum distance, among the calculated distances, was used to define the dominant emotion. It is important to note, that at that point, the results were calculated without using the adaptive part of the 
fuzzy method. The contribution of this component will be evaluated in section 3.5.

The results in table $1 \mathrm{a}$ and $1 \mathrm{~b}$ show that the proposed Fuzzy method had a better performance for both stages, and for both AV, and AV-AT models. At the same time, it provided us with an easily interpretable rule base, which allowed us to observe the underlying affect relations, in contrast to black box approaches such as the MLP.

Table 1.a. Stage 1 NRMSE and Dominant Emotion Accuracy for survey data.

\begin{tabular}{|c|c|c|c|c|c|c|c|c|c|c|c|}
\hline \multirow[t]{3}{*}{ Emotions } & \multicolumn{11}{|c|}{ NRMSE and Dominant Emotion Accuracy (STAGE1 SURVEY) } \\
\hline & \multicolumn{5}{|c|}{$\mathrm{AV}-\mathrm{AT}$} & \multicolumn{6}{|c|}{ AV } \\
\hline & FM & MLP & RBF & LNR & RT & FM & MLP & RBF & LNR & RT & D \\
\hline Flow & 16.30 & 17.92 & 18.05 & 21.10 & 19.89 & 22.28 & 23.08 & 22.52 & 24.90 & 25.83 & NA \\
\hline Excitement & 15.20 & 17.11 & 17.38 & 21.81 & 18.09 & 16.35 & 18.25 & 17.20 & 22.27 & 18.21 & NA \\
\hline Calm & 21.69 & 24.10 & 24.06 & 25.98 & 26.47 & 22.16 & 24.51 & 24.27 & 25.97 & 25.88 & NA \\
\hline Boredom & 16.09 & 17.50 & 17.09 & 21.88 & 19.42 & 17.03 & 17.70 & 17.82 & 21.98 & 19.46 & NA \\
\hline Stress & 18.73 & 20.18 & 19.90 & 22.15 & 23.57 & 20.20 & 21.57 & 21.42 & 23.19 & 23.80 & NA \\
\hline Confusion & 16.04 & 17.58 & 17.93 & 19.60 & 19.75 & 16.68 & 17.88 & 18.27 & 19.64 & 18.87 & NA \\
\hline Frustration & 17.97 & 19.31 & 19.85 & 21.67 & 21.36 & 19.78 & 21.43 & 21.72 & 22.63 & 22.65 & NA \\
\hline Neutral & 17.85 & 21.44 & 19.21 & 29.65 & 20.35 & 19.11 & 22.22 & 20.27 & 29.62 & 21.31 & NA \\
\hline Overall & 17.48 & 19.29 & 19.18 & 22.98 & 21.11 & 19.20 & 20.83 & 20.44 & 23.77 & & NA \\
\hline DEA & 66.94 & 64.44 & 63.68 & 62.01 & 59.69 & 60.56 & 54.24 & 58.82 & 56.25 & $\begin{array}{c}22.01 \\
51.77\end{array}$ & 54.17 \\
\hline
\end{tabular}

Table 1.b. Stage 2 NRMSE and Dominant Emotion Accuracy for survey data.

\begin{tabular}{|c|c|c|c|c|c|c|c|c|c|c|c|}
\hline \multirow[t]{3}{*}{ Emotions } & \multicolumn{11}{|c|}{ NRMSE and Dominant Emotion Accuracy (STAGE2 SURVEY) } \\
\hline & \multicolumn{5}{|c|}{ AV - AT } & \multicolumn{6}{|c|}{ AV } \\
\hline & FM & MLP & $\mathrm{RBF}$ & LNR & RT & FM & MLP & $\mathrm{RBF}$ & LNR & RT & $\mathrm{D}$ \\
\hline Flow & 19.14 & 20.87 & 20.71 & 22.87 & 22.79 & 20.79 & 21.89 & 21.89 & 23.76 & 24.40 & NA \\
\hline Excitement & 15.73 & 18.52 & 18.43 & 21.43 & 18.86 & 17.58 & 19.75 & 19.17 & 22.40 & 20.67 & NA \\
\hline Calm & 25.40 & 29.68 & 29.53 & 32.19 & 31.68 & 25.71 & 30.66 & 30.43 & 32.17 & 30.17 & NA \\
\hline Boredom & 19.76 & 21.52 & 21.65 & 24.00 & 24.93 & 20.28 & 21.07 & 21.29 & 24.03 & 23.73 & NA \\
\hline Stress & 20.12 & 22.17 & 21.82 & 23.04 & 24.64 & 20.75 & 21.74 & 21.75 & 23.19 & 24.38 & NA \\
\hline Confusion & 19.79 & 23.59 & 23.70 & 25.57 & 23.00 & 20.65 & 23.67 & 23.11 & 25.83 & 23.69 & NA \\
\hline Frustration & 18.15 & 18.88 & 19.31 & 22.22 & 22.27 & 19.95 & 19.57 & 19.61 & 22.80 & 21.53 & NA \\
\hline Neutral & 16.81 & 20.57 & 19.11 & 30.33 & 20.41 & 33.62 & 20.88 & 20.29 & 30.30 & 20.67 & NA \\
\hline Overall & 19.36 & 21.97 & 21.78 & 25.21 & 23.57 & 22.42 & 22.40 & 22.19 & 25.56 & 23.66 & NA \\
\hline DEA & 55.28 & 49.03 & 51.04 & 48.40 & 45.31 & 47.01 & 50.49 & 51.04 & 48.13 & 45.94 & 43.75 \\
\hline
\end{tabular}

In order to highlight the interpretability of the extracted fuzzy rule base we collocate the fuzzy rules obtained for flow and excitement.

If valence is positive, and arousal is high, and prediction is positive, then flow is very high. If valence is positive, and arousal is high, and outcome is better than expected, then excitement is very high.

As demonstrated by the classification results (Table 1), the AV-AT model appears to be significantly better in the first stage and marginally better to comparable in the second stage, for all systems, compared to the AV model. In addition, the NRMSE results for all classification systems are improved notably compared to the results in [33] where the Affective Trajectories hypothesis was proposed as the emotion modeling approach. The same applies for both stage 1 and stage 2 (prediction and outcome stages).

The online survey step was also required for the offline tuning of the system. With the help of the adaptive mechanism, the responses of a specific participant were used as desired 
changes to the original systems' predicted values. In this way, a new participant-specific fuzzy rule base was extracted, which was later used as a starting point for a personalized learning system. Finally the optimal position of the fuzzy sets centers for each of the input and output variables was calculated, with the help of the GA described in section 3.2.2.

\subsection{PERSONALIZED LEARNING SYSTEM}

The fuzzy mechanism along with the AV-AT model of emotionis utilized in order to construct a personalized learning system and enable the sugested methodology to be tested in realtime and in a specific context. The system's architecture provides a benchmark for AC systems to integrate the proposed fuzzy affect modeling approach in education, or other application contexts. The system's architecture is based on the two-stage emotion modeling approach, as seen in figure 3. This architecture comprises of two fuzzy classifiers, which utilize the fuzzy method described in section 3.2. The classifiers use the personalised fuzzy rule base extracted with the help of the online survey, which is unique for every user. Inputs for the classifiers are the prediction, arousal and valence elements for stage 1, and the outcome, valence, and arousal elements for stage 2 . The system is also inclusive of the adaptive mechanism as described in section 3.2.3 in order to provide the necessary changes to the fuzzy rule base when the user is not happy with the results. The output emotions for each stage comprise of the eight emotions used before. The system provides the appropriate feedback to improve the user's experience, based on the calculated values of the aforementioned emotions. In this research, the system is applied during educational sessions, which are divided into a number of different activities. A basic step-by-step implementation concerning one activity is described below. The same procedure is repeated for all consequent activities.

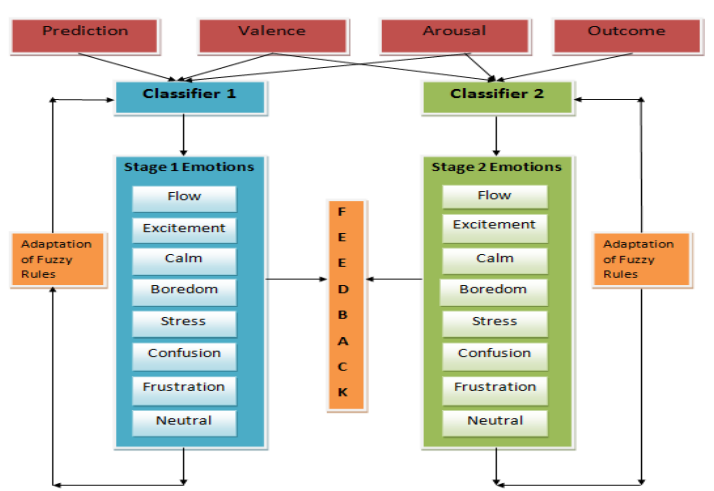

Fig. 3. Personalized learning system architecture. 
Before a new activity starts, the participant is asked to provide their prediction about the upcoming activity. The prediction along with the values of arousal and valence, which are also elicited from the participant, are given to classifier 1 which provides values for the eight target emotions. These values are presented to the user in order to reflect on their performance. If the user is not happy with the results, they have the option to provide new values for each of the eight emotions. The adaptive part of the system will then process these values, to make all the necessary changes to the rule base of the classifier. Given the calculated values of the eight emotions, the system presents tips and short motivational quotes to the user. When the activity ends, the user is asked to provide a value representing their evaluation of the outcome of the activity. This value, along with the arousal and valence values, are then fed into classifier 2 which will provide the necessary classification results. The system's feedback and adaptation is the same with stage 1 . In figure 4 , we can observe the affective trajectories of two users as provided by the system, over the course of a tutorial session consisting of 4 activities.
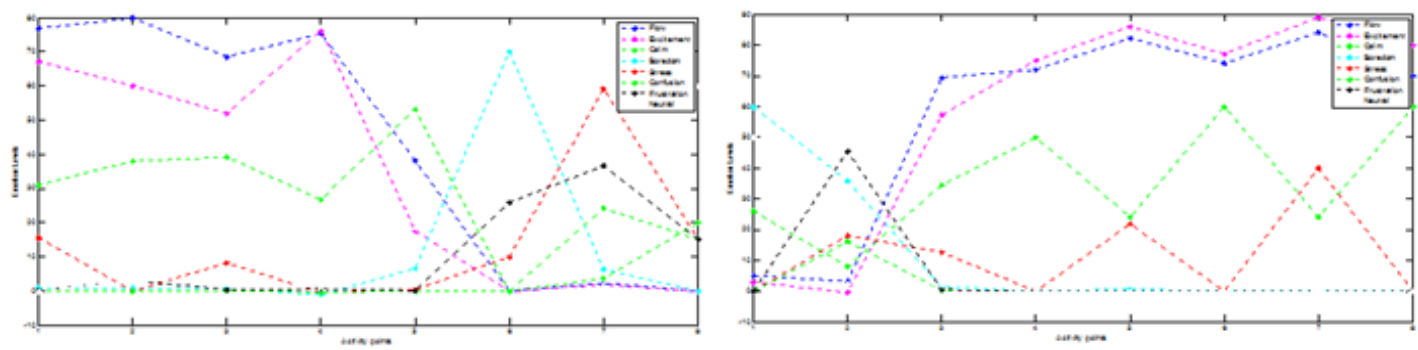

Fig. 4. System's output of students' affective trajectories during a tutorial session.

\subsection{MODEL EVALUATION}

Two practical experiments were designed and carried out in order to test the proposed approach. Twenty one participants, who had previously completed the online survey, participated in two tutorials comprising of two sessions each. The first tutorial was in the area of fuzzy logic, while the second covered a more general machine-learning topic, mostly focused on neural networks. Participants in both tutorials were divided into groups of three students, and they used their personal laptops on which the system was installed.

In the first tutorial, the participants were able to see the results and feedback of the system, and had the option to provide new values for the targeted emotion labels, to tune the system if they were not satisfied by the system's values. The values provided by the participants, along with the system's values were stored in order to be used for offline analysis. In the second tutorial session, the participants were asked to provide values of the target emotions at the beginning and at the end of every activity. During these sessions, they were not able to see the results, or the feedback of the system; as a result, the values they provided were not biased in any way. Nevertheless, the online adaptive part of the fuzzy method was still 
utilized in the background, making use of the values the participants provided, as desired changes to the system's responses. In both cases, the NRMSE was calculated for all emotion categories, along with the ability of the system to recognize the dominant emotion. Consistent with our previous experiments, we considered as dominant the emotion label with the highest value. Results for both practical sessions are presented in table 2 . In this table, values of the dominant emotion accuracy achieved when the AV model was used by applying the minimum distance method described in section 3.3, are also included.

Table. 2. NRMSE and Dominant Emotion Accuracy for practical experiments.

\begin{tabular}{lllll}
\hline Emotions & \multicolumn{4}{c}{$\begin{array}{c}\text { NRMSE and Dominant Emotion Accuracy (DEA) } \\
\text { Stage1 }\end{array}$} \\
& Practical Session 1 & Practical Session 2 & Practical Session 1 & Practical Session 2 \\
\hline Flow & 7.3253 & 11.8456 & 8.8728 & 13.4173 \\
Excitement & 8.3177 & 13.9371 & 7.1235 & 13.6475 \\
Calm & 9.3274 & 15.5236 & 8.1050 & 15.9639 \\
Boredom & 7.2292 & 10.0378 & 9.6106 & 11.9808 \\
Stress & 10.8370 & 11.8800 & 6.5552 & 9.9761 \\
Confusion & 6.1300 & 7.1484 & 9.6812 & 9.5869 \\
Frustration & 7.6439 & 9.6337 & 9.5817 & 8.3396 \\
Neutral & 5.5270 & 9.8717 & 8.6740 & 8.4263 \\
Overall & 7.7922 & 11.2348 & 8.5255 & 11.4173 \\
AV-AT DEA & $88.10 \%$ & $80.94 \%$ & $80.95 \%$ & $75.60 \%$ \\
AV DEA & $58.93 \%$ & $64.24 \%$ & $60.12 \%$ & $55.95 \%$ \\
\hline
\end{tabular}

It is evident from the results in Table 2 that the performance of the model massively outperforms the survey results for both practical sessions (Table 1). This is due to the adaptation process, which enabled the system to account for individual differences, which play a major role in the AV-AT emotion model in the same manner they do in the AT model [33]. Additionally, the AV-AT emotion model offers a better approach to recognizing the dominant emotion compared to the AV model for all cases and stages. This is clearer for stage 1, revealing the importance of the prediction element. The AV model scored around $60 \%$ for all stages and sessions, a percentage that was anticipated, if we consider that it is a stage independent model, since the emotion label used is dependant only on the arousal and valence values. In comparison with the adaptive version of the AT used in [33], the results are significantly better for both stages. In terms of the overall NRMSE the AT model scored 20.35 for the first, and 16.39 for the second stage respectively, which are worse compared to the results achieved by the AV-AT model. The difference in the results between the first and second tutorial is to be expected since the participants were not able to see the results of the system during the second tutorial and provided their own values for every case.

Once the participants had completed the tutorials they were formally debriefed, and they were also asked to provide their views concerning their experience of the system. It was noted that the participants' predictions were directly influenced by their mood (positive 
valence was related to a positive prediction), their familiarity with the subject (people more familiar with the subject made more positive predictions), and some personal characteristics such as an optimistic or a pessimistic stance. In addition, it was observed that the participants were happy to offer their predictions and evaluations concerning the activities. Providing their evaluations and predictions about the educational process made them more engaged rather than distracted.

Given the method's proven ability to model and monitor the affective trajectories of small groups of students, its true potential lies in the fact that it could be applied to perform largescale sentiment analysis and recognize the affective trajectories of larger groups of individuals. This computational model of emotion could provide a useful tool for performing sentiment analysis during the interaction of groups of people with social networks, and utilize the extracted results to provide estimates of their affective movement in time. Additionally, despite the fact that these tutorial sessions provide a measure of the method's performance, and useful ideas about its practical implementation, greater insight in the underlying emotion theory can be obtained by applying this method in social network data. This can be achieved by using a hybrid cloud, and monitoring the affective associations that millions of users make, concerning their predictions about the future, their evaluations of certain outcomes, and the emotion words they choose to describe their affective state. In order to further explore this affective methodology, and take advantage of this method's benefits in social network sentiment analysis, the necessary infrastructures should be able to process, analyze, and store big data. This ability is demonstrated in the following sections. This type of large-scale sentiment analysis could be beneficial in the developement of e-learning platforms and systems, and in the design of applications from different fields where the user's affect can be used as a factor in order to provide more focused feedback and tailored services.

\section{A HYBRID CLOUD SERVICE}

This section describes the hybrid cloud service able to integrate the proposed fuzzy computational model of emotion with sentiment and social network analysis, to ensure data can be processed and analyzed simultaneously on the Cloud. The core technologies include the following streams. Firstly, SQL and NoSQL databases to authenticate, query, process and store data. Secondly, the MapReduce and Spark frameworks to process large volume and velocity of data, and support parallel computing. Thirdly, the social network APIs that can fully translate the emotions of a large number of social network users into data analysis and visualization.. Fourthly, the multi-layered security adopted by Chang et al. [11] that 
can provide a robust security environment to withstand attacks from Trojans and viruses of 2013 known vulnerabilities. Multi-layered security consists of the integration of three major security technologies: (1) access control and firewall; (2) identity management and intrusion detection; and (3) encryption and decryption. The hybrid cloud is composed of three private clouds located at London, Leeds, and Southampton. [10]. The hardware infrastructure is presented in Table 3.

Table 3. Hardware infrastructure used for large-scale experiments and simulations.

\begin{tabular}{lllll}
\hline Location & $\begin{array}{l}\text { CPUs (average of } 3.0 \mathrm{GHz} \\
\text { per unit) }\end{array}$ & Memory (GB) & Storage (TB) & $\begin{array}{l}\text { Optimum Network speed } \\
(\mathrm{GBps})\end{array}$ \\
\hline London & 64 (control center) & $128 \mathrm{~GB}$ & $12 \mathrm{~TB}$ & 10 \\
Leeds & 32 & $64 \mathrm{~GB}$ & $8 \mathrm{~TB}$ & 1 \\
Southampton & 32 & $64 \mathrm{~GB}$ & $8 \mathrm{~TB}$ & 10 (March 2016)
\end{tabular}

The set up facilitates large-scale experiments and simulations to be undertaken in the Cloud in order to validate our approaches. The data has been owned and provided by Facebook as part of the Facebook developer agreement, covering the 2013-2014 period. The London based data center forms the control center because of its superior infrastructure and its location. Data is fed directly into the London control center where all other jobs for data processing can be distributed equally to each site. This can ensure each data center or cloud resource has a manageable amount of data. All the outputs of the analysis on the data were stored in the London and Leeds data centers for further analysis, extraction, and archiving of data.

Datumbox API is a Facebook API that can specializes in sentiment analysis and has been used to collect and analyze users' emotions [19]. To make the emotions previously used in this paper compliant to Facebook's sentiment analysis emotions in Table $4 \mathrm{a}$ have been categorized in a scale of 1 (the lowest) and 5 (the highest). The rationale for this is as follows. Frustration and stress are words of expressing negative feelings, and thus are rated as 1 . Confusion and boredom carry more aspects of negativity than the positive emotions and are rated as 2 . Neutral and calm represent words at an even scale and are rated as 3. Occasionally, calm is rated as 4 in situations that users are involved with accidents, natural disasters and unexpected incidents that may pose threats to personal safety. Flow is related to activities that Facebook users have been involved in and are willing to share.. The majority of these belong to positive categories and are thus rated as 4. In the data given for this experiment, $50 \%$ of "cools" have been used in neutral status and 50\% have been used in accidents, natural disasters or unexpected incidents, resulting in an equal split of ratings for 3 and 4. Excitement is related to events that make Facebook users delighted and are rated as 5. While using Datumbox API to process users' emotions, additional commands are written to ensure the smooth processing and analysis. The list of key functions is shown 
in Table 4b.

Table 4a. Categorization of the Facebook users' emotion matching our definition of emotion.

\begin{tabular}{lll}
\hline Emotions & Scale/rating & Related words \\
\hline Frustration & 1 & Frustrated; fed up; blow; annoyance; set back; upset \\
Stress & 1 & Stressful; stressed; pressure; pressurized; nervous; break down \\
Confusion & 2 & Confused; baffle; puzzled; no objectives \\
Boredom & 2 & Boring; apathy; dull; lack of activities \\
Neutral & 3 & Even; unbiased \\
Calm & 3 (or 4$)$ & Cool; steady; quiet but mindful; calmness \\
Flow & 4 & Moving; continuous; active \\
Excitement & 5 & Happy; delighted, blissful; over joy; feeling great \\
\hline
\end{tabular}

Table $4 \mathrm{~b}$. A list of key functions for processing sentiment analysis.

- $\operatorname{start}($ ): start the computational intelligence process

- job( ): start the job, with the control center in Leeds to kick of the process

- processing(London): processing data in London

- processing(Southampton): processing data in Southampton

- processing(Leeds): processing data in Southampton

- sentiment(All): analyze all sentiments

- sentiment(one): only analyze sentiments in rating 1

- sentiment(two): only analyze sentiments in rating 2

- sentiment(three): only analyze sentiments in rating 3
- sentiment(four): only analyze sentiments in rating 4

- sentiment(five): only analyze sentiments in rating 5

- results(one): display results in rating 1

- results(two): display results in rating 2

- results(three): display results in rating 3

- results(four): display results in rating 4

- results(five): display results in rating 5

- check(cool): to verify cool belongs to rating 3 or 4 .

- store(Leeds): store data in Leeds

- store(Southampton): store data in Southampton

- store(London): store data in London

- end( ): end the computational intelligence process

\section{LARGE SCALE EXPERIMENTS AND ANALYSIS OF OUR RESULTS}

In this section the analysis, and the results of all the large-scale simulations for our sentiment analysis, are described. In the first part, the execution time required to review each type of rating is demostrated. In the second part, the sentiment analysis for data on one million Facebook users is presented, and in the third part the correlation between our proposed approach and sentimental analysis on the Facebook users is discussed. Figure 5a shows the execution time for processing data for the data centers at London, Southampton and Leeds. London processes $50 \%$ of all the data, and Leeds and Southampton sites process $25 \%$ respectively. The execution time is consistent between London and Southampton, since the execution time completed for data processing in Southampton is close to half of the time required in London. There is also a similar execution time between London and Leeds. This due to the slower network speed and distance between the two locations.

Figure $5 \mathrm{~b}$ shows the execution time for sentiment analysis and presentation for all the ratings. Results are independent of the locations and all sites have results within a $1 \%$ difference. In the data provided by Facebook, sentiments that express a rating of 5 have the highest execution time, followed by rating 1,2 and then 3 and 4, which also follow a similar trend to the results in Figure 5a. Ratings with more "supporters" mean that a longer time is required to provide sentiment analysis and present results. Figure $5 \mathrm{c}$ shows the execution time for storing data at each site based on the mean values of the five experiments. London has the shorter execution time than Leeds to store data. Southampton has not been included 
since upgrades have been recently completed in March 2015.

Figure $5 \mathrm{~d}$ shows the total execution time for experiments in Figures $5 \mathrm{a}, 5 \mathrm{~b}$ and $5 \mathrm{c}$ between the London and Leeds data centers. Here it can be seen that there is a shorter execution time to complete all processing tasks in the London data center despite it processing $50 \%$ of the data. This is due to the experiments being carried out at this site and the site having a superior infrastructure. There is a $100 \%$ completion for all the data processing, analysis, and storage of data on both sites.

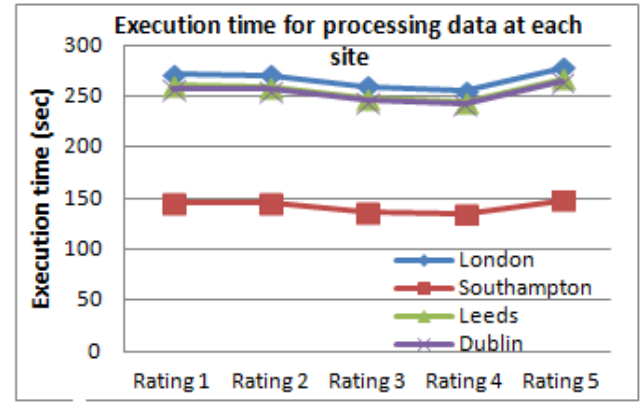

(a) Execution time for processing data at each site

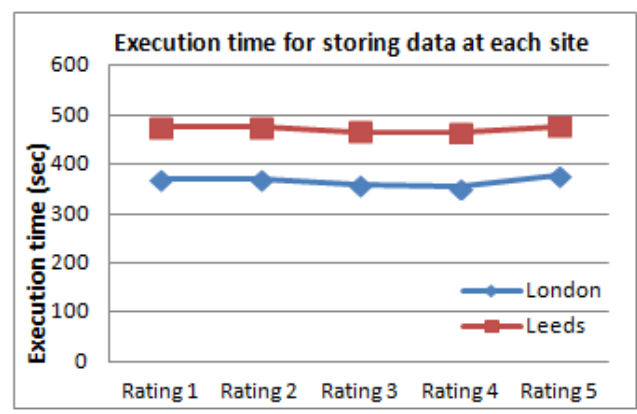

(c) Execution time for storing data at each site

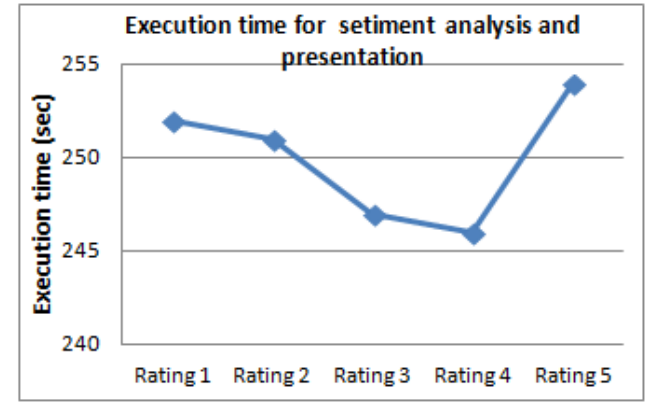

(b) Execution time for sentiment analysis and presentation

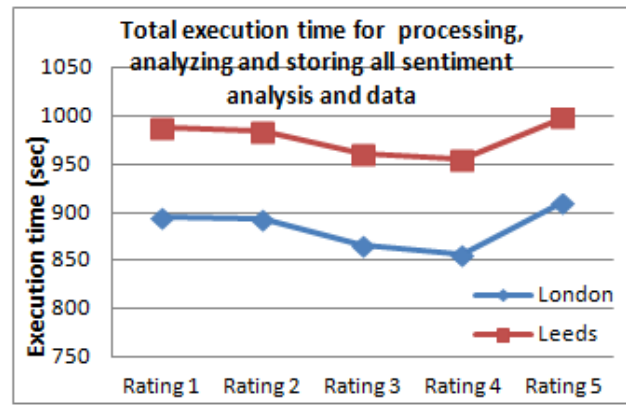

(d) Total execution time for processing, analyzing, and storing sentiment analysis and data

Fig. 5. Execution times.

Figure 6 shows results between our approach and Facebook's sentiment analysis. The comparison of the approaches does not meet $100 \%$ of matches due to various reasons such as vague status from the users, mixed feelings experienced by the users, and data being too large to handle. In our demonstration, results in Figure 5 show that the size of the data is not the reason for creating a mismatch. Hence, the likely causes are the vague status, which are hard to interpret correctly or the mixed feelings due to the nature of the events or the speed in which events have occured. While the status update is difficult to be captured fully for 1 million users, we can only rely on the availability of the disclosed data for us to perform analysis with the following steps. Firstly, all status updates have been categorized into five groups of rating. Secondly, a list has been processed that has been used for experiments for results in Figures 5a,b,c, and then after the end of experiments, another list has been processed to check whether all the status updates have been correctly categorized 
and analyzed to ensure there is a quality assurance process in place. The task is to check consistency between the use of our approach to categorize emotions into numerical ratings (the numerical and word mappings are shown in Table 4a) and the results of direct queries from Facebook Query Language (FQL) introduced by [13]. Sentiment analysis can be processed by numerical ratings in order to reduce processing time and complexities in dealing with large number of users [47, 38]. Although processing by numerical rating as specified in Table 4a can improve performance, it is important to check with results from FQL queries to ensure that our approach can get a high percentage of correct matches between words of emotion and the determined sentiments. Figure 6 shows that there is close correlation between these two approaches, with rating 5 having the highest match $(98.70 \%)$, followed by rating 1 (98.40\%), rating 2 (97.90\%), rating 4 (93.10\%) and rating 3 (92.50\%). When Facebook users have shown that they are either very upset or delighted, there is a clear distinction in their emotions that increases the matching percentage. However when the user has neutral feelings, it has become more challenging to find a match, although a matching of $92.50 \%$ can still be considered high.

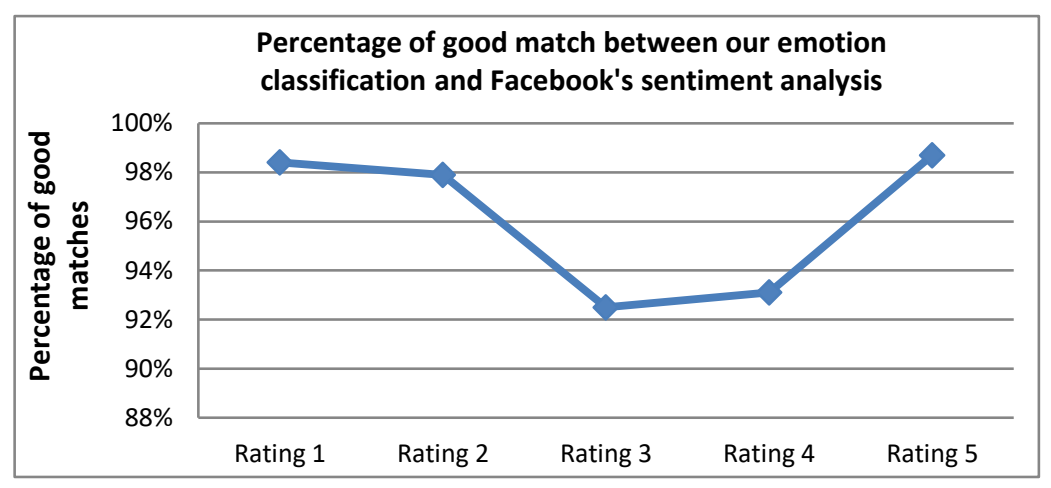

Fig. 6. Percentage of good match between our approach and Facebook's sentiment analysis.

\section{DISCUSSION}

In this section, we discuss the impact of our work and present comparison with other approaches. There are two groups of work to compare. The first group is the fuzzy logic approach to analyze and model emotions that comes under computer science and psychology disciplines. The second group is the use of cloud computing and big data processing for conducting large-scale sentiment analysis and social network analysis.

This paper presented a novel emotion representation, namely the AV-AT model. This new representation was tested through online and offline experiments, and the results illustrated that the AV-AT model was more effective in differentiating between the labels we choose to describe our affective state, when compared to the popular Arousal Valence (AV) representation [55], or the Affective Trajectories hypothesis [35]. The arousal valence 
model is extensively used by many state of the art AC systems, such as the ones proposed by Bustamante et al. and Soleymani et al. [7, 57]. The Affective Trajectories hypothesis was also used in recent $\mathrm{AC}$ research in order to facilitate the construction of affective computing systems [33]. As seen in figure 7, the AV-AT performs considerably better compared to the AV model in terms of Dominant Emotion Accuracy, and compared to the AT model in terms of the NRMSE. These findings support the potential usefulness of the model in the hands of AC researchers in order to use sets of emotions, which better describe their user's affective state, when compared to other approaches. The AV-AT model offers more flexibility in the choice of target emotions by harnessing the benefits of a two-stage, and 3D emotion representation. Moreover, it facilitates a deeper understanding of emotional processes, since it was driven by affect-recognition purposes, instead of imitating human emotion for virtual agents, such as the computational models of emotion reviewed by Marcella et al. [42] and Kowalcuzk et al. [36].
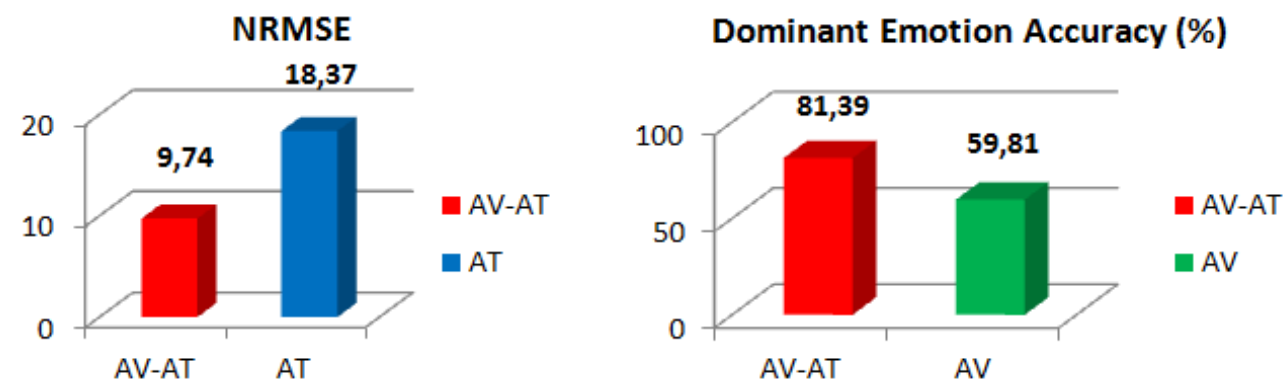

Fig. 7. AV-AT model of emotion vs. AV and AT models.

This paper's affect-modeling approach can be applied in various contexts. This can be achieved by modifying the set of target emotions. In an affective driving application for example, the system could use a set of emotions similar to the one used by Nasoz et al. [44]. This set comprised of six emotions namely panic, frustration, anger, boredom, fatigue, and fear. In affective gaming a set of emotions as the one used in [41] can be utilised, whereas in an affective learning application a set of emotions such as the one described in this paper would be appropriate. The only limitation to be considered is the requirement for discrete points in time in order to obtain estimates of the basic elements of prediction and outcome.

Literature informs us that, our affective state correlates strongly with changes to our physiology. More specifically, in Rainville et al.'s work, in 2006, it was shown that the heart rate of an individual increases when positive stimuli is presented to them [52]. In addition, in [43] the relation of changes in skin temperature with the negative or positive valenced affective state of an individual was explored. In [20] we can observe the close association between arousal levels and the galvanic skin response signal. From the above we conclude that estimates of arousal and valence elements can be automatically extracted 
with the use of the relevant physiological sensors. This can be achieved with the help of non-obtrusive wearable devices such as the Autosense, the Empatica E3, or E4 sensors and other available sensors. This sensor-specific information can be combined with estimates of the prediction and outcome elements. These elements, as illustrated in section 3.5, can be extracted from a combination of the user profile, and other contextual information. Therefore, a two-leveled system such as the one proposed in [41] that utilizes the AV model, could be used in order to fully automate the emotion recognition process.

Concerning the comparison of the proposed approach in the utilization of cloud computing and big data processing for conducting large-scale sentiment analysis, and social network analysis, we discuss the following observations in respect to relevant work. Ortigosa et al. [45] use a Facebook API, SentBuk, to collect and analyze user data. In their work, they only use three types of emotions: positive, neutral and negative, with $66.89 \%$ of users being positive, thus representing a biased selection of their choice. They explain how their work is relevant to e-learning using a case study. However, they do not use cloud intelligence and some technical details of their approach are vaguely described. Krishna et al. [37] have illustrated their rationale, steps, and results from their experiments to demonstrate that Facebook sentiment analysis can be conducted on cloud computing. Nevertheless, they do not provide details about the types of data they have dealt with, nor details of the cloud resources used. Ren [53] presents one of the first papers in this area. They explain the concepts of using a fuzzy logic based system to collect and analyze users' emotions in the cloud. There are measurements on different types of emotions collected and translated to the stakeholders. Although there are interesting concepts discussed, there is no information about how to replicate their approach using standard APIs from social networks such as Facebook or Twitter, or how to set up and measure users' data intelligently, and whether such data is derived from their own case study.

\section{CONCLUSION AND FUTURE WORK}

This paper introduced a methodology for incorporating emotion in the design of intelligent computer systems, and explored its applicability and performance, through currying out a series of online and offline experiments. The approach presented, initially establishes the mixed AV-AT emotion model. In order for this model to be successfully utilized, an adaptive fuzzy modeling method was implemented, which used optimized parameters with the help of a GA. A framework and basic architecture was proposed, which integrates the suggested approach, so that it can be utilized by affective computing systems. Moreover, an online personalized learning system was developed to evaluate the 
performance of the suggested affect modeling methodology in a real setting, while at the same time promoting student learning and engagement within modern pedagogical contexts.

We have also demonstrated a cloud computational intelligence infrastructure, which can integrate the suggested emotion modeling approach, and explain a list of sentiment analysis functions. This was achieved by conducting large-scale experiments carrying out data processing, sentiment analysis, and storage on data comprising of one million Facebook users. The proposed emotion modeling approach can be used as part of a cloud intelligence framework through the use of hybrid cloud services. Data centers in London, Southampton, and Leeds that have been used to validate our approach. Explanations for our research impact have been justified to ensure that our work is unique and significant. Contributions for big data processing were explained to ensure that our work could bridge the gap between theory and practice. Comparisons with other approaches were discussed to reflect the quality of our work and allow us to think of future developments of the work.

The main contributions of our research can be summarized as follows:

- A novel emotion modeling methodology is proposed for incorporating human emotion as part of intelligent computer systems.

- A new mixed representation of emotion called the AV-AT model is presented offering high recognition accuracy and enabling flexibility in choosing suitable sets of emotions.

- The adaptive fuzzy method presented, achieved a satisfactory classification performance compared to other well-known ML approaches, while at the same time retaining a high degree of interpretability of the underlying affect relations through its use of fuzzy rules. Moreover, it demonstrated the importance of individual differences in selecting emotion labels based on the structural elements of the AV-AT model.

- A personalized learning system was developed, specifically designed for assisting students in the context of PBL pedagogical framework that has been tested successfully in two practical tutorial sessions.

- Research directions were presented for applying this methodology in various contexts such as driving and gaming, and towards the parallel use of physiological sensors to facilitate the emotion recognition process.

- We demonstrated cloud intelligence and provided evidence of the ability and effectiveness of a large-scale deployment. Our hybrid cloud intelligence service processed and performed sentiment analysis and stored the outputs, with competitive execution times at all sites. 
- The proposed computational intelligence based emotion modeling approach was used to implicitly classify emotion states, and achieved a high percentage of matching with Facebook's sentiment analysis.

By providing a novel computational methodology to represent and model emotion, we aim to enhance our understanding of the incorporation of emotion in the design of intelligent computing systems, resulting in the improvement of services provided by those systems to their users. Future work will involve the modification of our approach to account for the transition probabilities between affective states. We aim to achieve this by using dynamic modeling tools, such as the Fuzzy Cognitive Map (FCM) [56] methodology. Future developments of the work will also include analysis of more up-to-date, larger scale, and longer-term analysis of user data, along with the deployment of state of the art bioinspired optimization algorithms in order to improve specific parameters of the constructed fuzzy model.

\section{REFERENCES}

[1] K. Almohammadi, Bo Yao and H. Hagras, "An interval type-2 fuzzy logic based system with user engagement feedback for customized knowledge delivery within intelligent E-learning platforms," Fuzzy Systems (FUZZ-IEEE), IEEE International Conference on, 6-11 July, 2014, pp.808-817.

[2] L.F. Barrett,B. Mesquita, \& M. Gendron, (2011). Context in emotion perception. Current Directions in Psychological Science, 20(5), 286-290.

[3] H. S. Barrows, "Problem-based learning in medicine and beyond: A brief overview", New Directions for Teaching and Learning, vol. 68, no. 3, 1996.

[4] D. Bernardo, H. Hagras \& E.P.K. Tsang, An Genetic Type-2 fuzzy logic based system for financial applications, modeling and prediction, Proceedings, IEEE International Conference on Fuzzy Systems (FU-IEEE), Hyderabad, India, 7-10 July 2013.

[5] J. Bezdek, Pattern Recognition with Fuzzy Objective Function Algorythms. New York: Plenum, 1981.

[6] M.M. Bradley, P.J. Lang (2010). Affective Norms for English Words (ANEW): Instruction manual and affective ratings. Technical Report C-2, Gainesville, FL.The Center for research in Psychophysiology, University of Florida.

[7] P.A Bustamante, N.L. Celani, M.E. Perez and O.Q. Montoya (2015, August). Recognition and regionalization of emotions in the arousal-valence plane. In 37th Annual International Conference of the IEEE Engineering in Medicine and Biology Society (EMBC), pp. 6042-6045. IEEE.

[8] R.A. Calvo, \& S. D'Mello (2010). Affect detection: An interdisciplinary review of models, methods, and their applications. Affective Computing, IEEE Transactions on, 1(1), 18-37.

[9] M., Chan, E., Campo, D., Estèvem and J. Y., Fourniols, (2009). Smart homes-current features and future perspectives. Maturitas, 64(2), 90-97.

[10] V., Chang. (2015). Towards a Big Data system disaster recovery in a Private Cloud. Ad Hoc Networks, 35, 65-82.

[11] V., Chang, Y. H., Kuo and M., Ramachandran. (2016). Cloud computing adoption framework: A security framework for business clouds. Future Generation Computer Systems, 57, 24-41.

[12] V., Chang, R.J., Walters, and G.B., Wills, (2016). Organisational sustainability modeling-An emerging service and analytics model for evaluating Cloud Computing adoption with two case studies. International Journal of Information Management, 36(1), 167-179.

[13] Chang, V. (2016). A Cybernetics Social Cloud. Journal of Systems and Software, in press.

[14] Cook, Diane J., Juan C. Augusto, and Vikramaditya R. Jakkula. "Ambient intelligence: Technologies, applications, and opportunities." Pervasive and Mobile Computing 5.4 (2009): 277298.

[15] S. Craig, A. Graesser, J. Sullins, and B. Gholson, “Affect and Learning: An Exploratory Look into the Role of Affect in Learning," Journal of Educational Media, vol. 29, no. 3, 2004, pp. 241-250. 
[16] M. Csikszentmihalyi, Flow: the psychology of optimal experience, New York, NY: Harper Row, 1990.

[17] W. A. Cunningham and P. D. Zelazo, “The Development of Iterative Reprocessing: Implications for Affect and its Regulation," In Developmental Cognitive Social Neuroscience. P.D. Zelazo, M. Chandler and E. Crone, Eds. New York, NY: Psychology Press, 2009, pp. 81-98.

[18] W. A. Cunningham, K. A., Dunfield and P. E. Stillman, "Emotional States from Affective Dynamics," Emotion Review vol.5, no. 4, 2013, pp. 344-355.

[19] Datum Box, Machine Learning API, accessible http://www.datumbox.com/machine-learning-api/ on 20 March, 2016.

[20] M.E. Dawson, A.M. Schell and D.L. Filion,(2007) The electrodermal system, In Cacioppo, J. T., Tassinary, L. G., Berntson, G. (eds), Handbook of Psychophysiology. Cambridge University Press, Cambridge.

[21] P. Dillenbourg, Collaborative Learning: Cognitive and Computational Approaches. Advances in Learning and Instruction Series. New York, NY: Elsevier Science, Inc, 1999.

[22] S.K. D’Mello, and R.A. Calvo (2013) 'Beyond the Basic Emotions: What Should Affective Computing Compute?'. in S. Brewster, Bødker, S., and Mackay, W. (eds.) Extended Abstracts on the ACM SIGCHI Conference on Human Factors in Computing Systems (CHI 2013), pp. 22872294.

[23] F.Doctor, H. Hagras, V. Callaghan, 'A Fuzzy Embedded Agent-Based Approach for Realizing Ambient Intelligence in Intelligent Inhabited Environments," IEEE Transactions on Systems, Man and Cybernatics- Part A: Systems and Humans, vol. 35, no. 1, January 2005, pp. 55-56.

[24] F. Doctor, C. Syue, Y. Liu, J. Shieh, R. Iqbal, Type-2 fuzzy sets applied to multivariable selforganizing fuzzy logic controllers for regulating anesthesia, Applied Soft Computing, Volume 38, January 2016.

[25] P. Ekman and W. V. Friesen "Unmasking the Face: A Guide to Recognizing Emotions from Facial Expressions,” Englewood Cliffs, New Jersey, NJ: Prentice-Hall, 1975.

[26] E. Elbeltagi, T. Hegazy, \& D. Grierson (2005). Comparison among five evolutionary-based optimization algorithms. Advanced engineering informatics, 19(1), 43-53.

[27] M. Gendron, \& L.F. Barrett,(2009). Reconstructing the past: A century of ideas about emotion in psychology. Emotion Review, 1, 316-339

[28] A.C. Graesser, P. Chipman, B.C. Haynes \& A. Olney,(2005) AutoTutor: An intelligent tutoring system with mixed-initiative dialogue. IEEE Transactions in Education, 48, 612-618

[29] Hashem, I. A. T., Yaqoob, I., Anuar, N. B., Mokhtar, S., Gani, A., \& Khan, S. U. (2015). The rise of "big data" on cloud computing: Review and open research issues. Information Systems, 47, 98 115.

[30] J. J., Huang, S. J., Yang, Y. M., Huang and I. Y., Hsiao, (2010). Social Learning Networks: Build Mobile Learning Networks Based on Collaborative Services. Educational Technology \& Society, 13(3), 78-92.

[31] R. Iqbal, A. James, L. Payne, M. Odetayo, H. Arochena (2008), Moving to Activity-led Learning in Computer Science, In proceedings of iPED 2008, 3rd International Conference, pp.125-131, UK. ISBN: 978-1-84600-0218

[32] R. Iqbal, F. Doctor, M. Romero, A. James (2013), Activity-Led Learning Approach and Group Performance Analysis Using Fuzzy Rule-Based Classification Model, Proceedings of the 17th International Conference on CSCW in Design, IEEE, pp., 599-606.

[33] C. Karyotis, F. Doctor, R. Iqbal, and A. James, (2015) An Intelligent Framework for Monitoring Students Affective Trajectories Using Adaptive Fuzzy Systems, Proceedings, IEEE International Conference on Fuzzy Systems, 2-5 August 2015, Istanbul, Turkey.

[34] A. Kazemzadeh, S. Lee and S. Narayanan, "Fuzzy Logic Models for the Meaning of Emotion Words,” IEEE Computational Intelligence Magazine vol.8, issue 2, May 2013, p.34-49.

[35] T. Kirkland and W. A. Cunningham, "Mapping Emotions Through Time: How Affective Trajectories Inform the Language of Emotion," Emotion, vol. 12, no. 2, 2012, pp. 268-282.

[36] Z. Kowalczuk, and M. Czubenko (2016) Computational Approaches to Modelling Artificial Emotion-An Overview of the Proposed Solutions. Frontiers in Robotics and Artificial Intelligence [online] 3(21). available from 〈http://journal.frontiersin.org/article/10.3389/frobt.2016.00021/full> [10 July 2016]

[37] P. V., Krishna, S., Misra, D., Joshi and M. S., Obaidat. (2013, May). Learning automata based sentiment analysis for recommender system on cloud. In IEEE 2013 International Conference on Computer, Information and Telecommunication Systems (CITS), pp. 1-5. 
[38] Leung, C. W., Chan, S. C., \& Chung, F. L. (2006, August). Integrating collaborative filtering and sentiment analysis: A rating inference approach. In Proceedings of the ECAI 2006 workshop on recommender systems (pp. 62-66).

[39] K. Lindquist, Emotion Review 2013 5: 356 Kristen A. Lindquist Model Emotions Emerge from More Basic Psychological Ingredients: A Modern Psychological Constructionist Published by:SAGE doi: 10.1177/1754073913489750 Emotion Review October 2013 vol. 5no. 4 356-368

[40] C. Lisetti, F. Nasoz, C. LeRouge, O. Ozyer, and K. Alvarez, (2003) Developing multimodal intelligent affective interfaces for tele-home health care. Internarional Journal of Human-Computer Studies, 59(1-2), 245-255.

[41] R.L. Mandryk, M.S. Atkins, (2007) A Fuzzy Physiological Approach for Continuously Modeling Emotion during Interaction with Play Technologies. International Journal of Human-Computer Studies, 65, 329- 347.

[42] S. Marsella, J. Gratch, and P. Petta, (2010) Computational Models of Emotion. in A Blueprint for Affective Computing-A Sourcebook and Manual. ed. by Scherer, K. R., Bänziger, T., and Roesch, E. Oxford: Oxford University Press, 21-46

[43] R.A. McFarland, (1985) Relationship of skin temperature changes to the emotions accompanying music. Biofeedback and Self-regulation, 10(3), 255-267.

[44] F. Nasoz, C.Lisetti,and A.Vasilakos A. (2010) Affectively intelligent and adaptive car interfaces. Information Sciences, 180, 3817-3836. A.,

[45] Ortigosa, J. M., Martín and R. M. Carro. (2014). Sentiment analysis in Facebook and its application to e-learning. Computers in Human Behavior, 31, 527-541.

[46] A. Ortony, \& T.J. Turner, (1990). What's basic about basic emotions? Psychological Review, 97, 315-331.

[47] Pang, B., \& Lee, L. (2005, June). Seeing stars: Exploiting class relationships for sentiment categorization with respect to rating scales. In Proceedings of the 43rd Annual Meeting on Association for Computational Linguistics (pp. 115-124). Association for Computational Linguistics.

[48] B. Pang and L. Lee. Opinion mining and sentiment analysis. Foundations and Trends in Information Retrieval, 2(1-2):1-135, 12008

[49] R. Picard, (1995) Affective computing Technical Report no. 321. Cambridge: MIT Media Laboratory.

[50] R. Picard, (1997) Affective Computing. Cambridge, MA: MIT Press. J.,

[51] Preece and D., Maloney-Krichmar. (2003). Online communities: focusing on sociability and usability. Handbook of human-computer interaction, 596-620.

[52] P. Rainville, A. Bechara, N. Naqvi and A.R. Damasio, (2006) Basic emotions are associated with distinct patterns of cardiorespiratory activity. International Journal of Psychophysiology, 61(1), 518. F.,

[53] Ren (2010). From cloud computing to language engineering, affective computing and advanced intelligence. International Journal of Advanced Intelligence, 2(1), 1-14.

[54] J. A. Russell, (1980) A Circumplex Model of Affect. Journal of Personality and Social Psychology, 39(6), 1161-1178.

[55] J. A. Russell, (2003). Core affect and the psychological construction of emotion. Psychological Review, 110, 145-172.

[56] J. L. Salmeron, (2012). Fuzzy cognitive maps for artificial emotions forecasting. Appl. Soft Comput, 12(12), 3704-3710.

[57] M. Soleymani, S. Asghari-Esfeden, Y. Fu, \& M. Pantic (2016). Analysis of EEG signals and facial expressions for continuous emotion detection. IEEE Transactions on Affective Computing, 7(1), $17-28$

[58] Tan, C., Lee, L., Tang, J., Jiang, L., Zhou, M., \& Li, P. (2011, August). User-level sentiment analysis incorporating social networks. In Proceedings of the 17th ACM SIGKDD international conference on Knowledge discovery and data mining (pp. 1397-1405). ACM.

[59] J.L.Tsai, J.Y. Louie, E.E. Chen, \& Y. Uchida, (2007). Learning what feelings to desire: Socialization of ideal affect through children's storybooks.Personality and Social Psychology Bulletin, 33(1), 17 30 .

[60] L. X. Wang, "The MW method completed: A flexible system approach to data mining," IEEE Transactions Fuzzy Systems, vol. 11, no. 6, 2003, pp. 768-782.

[61] S. Wilson-Medhurst,I. Dunn, P. White, R. Farmer and D. Lawson (2008).Developing Activity Led Learning in the Faculty of Engineering and computing at Coventry University through a continuous improvement change process. Proceedings of Research Symposium on Problem Based Learning in Engineering and Science Education, Aalborg, Denmark, June 30 - July 1, 2008. 
[62] D. Wu, et al., "Using Physiological Signals: Virtual Reality Stroop Task," IEEE Transactions on Affective Computing vol. 1, no. 2, 2010, pp. 109-118.

[63] D. Wu, (2012) Fuzzy sets and systems in building closed-loop affective computing systems for human-computer interaction: Advances and new directions, IEEE World Congress on Computational Intelligence, Brisbane, Australia

[64] L.A. Zadeh, (1965). Fuzzy sets. Information and Control 8, pp. 338-353.

[65] Z. Zeng, M. Pantic, G.I. Roisman, and T.S. Huang, "A Survey of Affect Recognition Methods: Audio, Visual, and SpontaneousExpressions," IEEE Trans. Pattern Analysis and Machine Intelligence, vol. 31, no. 1, pp. 39-58, Jan. 2009. 